\section{Celebrating the Birth of Copernicus}

by our Astronomy Correspondent

Ir is fortunate for the organizers of celebratory conferences that the quincentenary of the birth of Copernicus comes at a time when the study of the solar system and planets is in a healthier state than ever before, even though the end of the Apollo programme has left many selenologists less cheery than is seemly for scientists with so much unanalysed data still up their sleeves. After all, only a few weeks ago an article in Science reported the bemoanings of staff at the Manned Spacecraft Center, who apparently have more lunar material than they know what to do with. Had the Copernicus quincentenary occurred only fourteen years ago, before the Lunik flights that is, the celebratory symposium held by the Royal Society and the Royal Astronomical Society in London last week would have dealt with the work of a few dedicated scientists, far removed from the modish astronomy of the day, struggling to glean what they could by peering at objects which from the Earth are only a few seconds of arc in diameter (for example, Mars, 25 arc s) or at best 30 minutes of arc in diameter (the Moon).

Things are different now, as Professor S. K. Runcorn (University of Newcastle upon Tyne) made clear in his review of knowledge of the Moon, which was founded for the most part on information which has come from the Apollo missions and the preceding Orbiter series of automatic lunar satellites (the Orbiters relayed back to the Earth enough detailed photographs of the Moon to keep selenologists busy for years). For example, Professor Runcorn suggested that the moonquakes which have been identified by the seismic network on the Moon as occurring at the edges of the circular maria may arise as a result of the slow sinking of material into the Moon at these locations. According to Professor Runcorn, the existence of this slowlysettling material is suggested by the presence of the gravity anomalies at the circular maria (the mascons, identified by analysis of the motions of Orbiter spacecraft). He suggested that these mascons arise after the circular mare basin has been punched out of the surface by the impact of a meteorite, and after the basin has been filled with lava from below. The solidification of the lava pool leads to a fall in the level of the surface by about $2 \mathrm{~km}$, and it is the further molten material which has welled up from below to fill this $2 \mathrm{~km}$ which is the cause of the anomalous gravity, while the gradual sinking of this circular plug of material gives rise to some of the moonquakes.

But Professor Runcorn could offer no evidence bearing on the question of the origin of the Moon, which, he said, is still largely a matter of taste, although he prefers the view that the Moon accreted from a cloud of dust around the Earth.

Venus and Mars were dealt with by Professor R. Goody (Harvard University) who said that some kind of consensus is now emerging on several topics to do with these planets as a result of the American and Soviet space programmes, ground-based observations, and theoretical work by geophysicists. He said that the investment in this work of a small proportion of the budgets of the American, Soviet and European space programmes will ensure continuing revolutionary discoveries.

Compared with the Earth, Mars and probably also Venus are gravitationally rough, indicating the existence of strong tectonic processes. Professor Goody recited the important discoveries on Mars made by Mariner 9 , which include the largest volcano in the solar system (Nix Olympica, which seems to be at least $10 \mathrm{~km}$ high, and perhaps even higher), an enormous canyon with dendritic features (Coprates, which among other reasons is odd because there is no sign of any debris which might have been expected to be left behind), and features resembling desert washes which must have arisen by the flow of a liquid over the Martian planes.

The cameras also snapped one of the Martian moons, Phobos, which, of course, can have no atmosphere or internal processes to wipe its surface clean of craters because of its small size. Its crater density, a hundred times that of the Martian surface in general, should therefore be a useful calibration mark for studies in which the ages of Martian surface features are estimated from the density of craters.

As a meteorologist, Professor Goody was obviously not too disturbed by the dust storm which at first hid the Martian surface from the cameras. He conjectured that a mechanism not unlike that of a tropical hurricane could pump the atmosphere of Mars full of dust in a matter of days, and said that some of the photographs towards the end of the storm show features reminiscent of a terrestrial cold front. Professor Goody concludes from his study of the Martian atmosphere that seas might have existed there at an earlier epoch, so that Mars ought to be examined for fossils.

Jupiter and Saturn have not been studied by space probes, although Pioneer 10 is now across the asteroid belt and should reach Jupiter toward the end of this year, so Professor R. Hide (Meteorological Office) was limited to ground-based information in his talk, although the radio emission from Jupiter is a useful phenomenon which no other planet can match. It seems that the emission can be divided into three classes-thermal emission from the disk, decimetre radiation which comes from an area much larger than the disk and is assumed to have a synchrotron origin, and decametre radiation which appears in bursts apparently controlled by the position of the satellite Io and which comes from areas much smaller than the disk.

Professor Hide is also a meteorologist and much of his talk was a discussion of the atmospheres of Jupiter and Saturn, in particular the Red Spot of Jupiter which has a history going back three centuries, whereas with other similarsized features in the Jovian atmosphere seem to come and go in a matter of weeks. Professor Hide still seems to favour the view that the Red Spot is a Taylor column depending for its existence on an interaction between the atmosphere and some kind of feature on the surface of the planet (or between the atmosphere and a solid object floating in it).

Copernicus's own field of planetary motions was discussed by Dr G. A. Wilkins (HM Nautical Almanac Office). In particular Dr Wilkins discussed the strange relations which occur between the periods of the outer planets, and between the periods of their satellites. For example, five periods of Jupiter are equal to 2.013 periods of Saturn, three periods of Saturn equal 1.052 periods of Uranus, and two periods of Uranus equal 1.020 periods of Neptune. Similar commensurabilities occur among the satellites of Jupiter and Saturn (indeed, in the case of Saturn the relations extend to some features of the ring system). A full explanation of these phenomena is still not availablequestions such as how the regularities arose, and whether they strengthen the system or will eventually lead to the system breaking up, need to be answered-but it seems that tidal friction must be the chief mechanism.

Dr Wilkins also discussed Ovenden's "Principle of Least Interaction Action" by means of which Ovenden has come to some conclusions about the nature of a missing planet in the asteroid belt (Nature, 239, 508 ; 1972), and although the full details have yet to be published Dr Wilkins feels that Ovenden's principle may turn out to be useful in planetary mechanics. 